Präoperative Kurzzeitradiotherapie kombiniert mit volldosierter Langzeitchemotherapie beim lokal fortgeschrittenen Rektumkarzinom. Das Beste aus zwei Welten

Guckenberger, M

DOI: https://doi.org/10.1007/s00066-014-0792-0

Posted at the Zurich Open Repository and Archive, University of Zurich ZORA URL: https://doi.org/10.5167/uzh-110317

Journal Article

Published Version

Originally published at:

Guckenberger, M (2015). Präoperative Kurzzeitradiotherapie kombiniert mit volldosierter Langzeitchemotherapie beim lokal fortgeschrittenen Rektumkarzinom. Das Beste aus zwei Welten. Strahlentherapie und Onkologie, 191(3):285-287.

DOI: https://doi.org/10.1007/s00066-014-0792-0 


\title{
Präoperative Kurzzeitradiotherapie kombiniert mit volldosierter Langzeitchemotherapie beim lokal fortgeschrittenen Rektumkarzinom
}

\author{
Das Beste aus zwei Welten?
}

Matthias Guckenberger

Online publiziert: 26. November 2014

(C) Springer-Verlag Berlin Heidelberg 2014

Hintergrund Der Einsatz multimodaler Therapie beim lokal fortgeschrittenen Rektumkarzinom reduziert die Lokalrezidivrate auf $<10 \%$, so dass heute vor allem die Fernmetastasierung das Überleben der Patienten limitiert. Es ist daher das Ziel aktueller Studienkonzepte, die systematische Therapiekomponente $\mathrm{zu}$ intensivieren, ohne durch eine gesteigerte Toxizität die Erfolge der Lokaltherapie neoadjuvante Radiotherapie (RT) und TME - zu gefährden. Die aktuelle Studie kombiniert eine sequenzielle Kurzzeitbestrahlung und eine FOLFOX-Chemotherapie.

Methode In einer monozentrischen prospektiven Phase-IIStudie wurden 79 Patienten mit lokal fortgeschrittenem (cT3 oder cT4), histologisch gesichertem Rektumkarzinom mit einer Kurzzeit-RT mit 5x5 Gy und nach einem Intervall von maximal 12 Arbeitstagen zusätzlich mit 4 Zyklen FOLFOX behandelt [1]. Die operative Therapie war 13-18 Wochen nach Beginn der RT vorgesehen. Eine postoperative Chemotherapie war optional. Primärer Endpunkt der Studie war das Downsizing der T-Kategorie und sekundärer Endpunkt die Rate an gastrointestinaler Toxizität vom Grad $\geq 3$.

Ergebnisse Die präoperative FOLFOX-Chemotherapie konnte bei $95 \%$ der Patienten vollständig appliziert werden.

Originalbeitrag: Myerson RJ, Tan B, Hunt S et al (2014) Five fractions of radiation therapy followed by 4 cycles of FOLFOX chemotherapy as preoperative treatment for rectal cancer. Int $\mathrm{J}$ Radiat Oncol Biol Phys 88(4):829-836

Prof. Dr. M. Guckenberger, M.D. ( $₫)$

Department of Radiation Oncology,

University Hospital Zurich (USZ),

Rämistrasse 100,

8091 Zürich, Schweiz

E-Mail: Matthias.Guckenberger@usz.ch
Insgesamt 76 von 79 Patienten wurden lokal am Rektumkarzinom operiert. Die R1-Resektionsrate betrug 5\%. Ein T-Downstaging wurde bei $71 \%$ der Patienten erzielt. Bei $25 \%$ der Patienten konnte eine pathologisch komplette Remission (pCR) erreicht werden. Eine Toxizität der gastrointestinalen bzw. hämatologischen Kategorie vom Grad $\geq 3$ erlitten 9\% bzw. 27\% der Patienten. Nach einer durchschnittlichen Nachbeobachtungszeit von 26 Monaten wurde bei 3 Patienten ein Lokalrezidiv beobachtet. Ein Patient verstarb an einer Darmperforation als Spättoxizität.

Schlussfolgerung der Autoren Das Tumoransprechen sowie die Verträglichkeit des Studienprotokolls erscheinen im Vergleich zur präoperativen simultanen Radiochemotherapie vielversprechend.

\section{Kommentar}

Nachdem in zwei randomisierten Studien keine Unterschiede der Ergebnisse nach präoperativer Kurzzeit-RT und 5-FU-basierter Langzeit-RCHT gefunden wurden [2, 3], stellt das aktuelle Protokoll eine konsequente Weiterentwicklung der Kurzzeitstrategie dar. Methodik, Verträglichkeit und Effektivität werden im Folgenden diskutiert.

In der Studie der Washington University wurden modernste Bestrahlungskonzepte angewendet, die bisher noch keinen Einfluss in die großen internationalen Multizenterstudien gefunden hatten. Alle Patienten wurden mit intensitätsmodulierter Strahlentherapie (IMRT) und mit einer durch Cone-Beam-CT geführten Strahlentherapie (IGRT) behandelt. Das Dosis- und Zielvolumen Konzept verwendete einen simultan integrierten Boost (SIB) mit einer Dosisdeeskalation auf 5x4 Gy im elektiven regionären Lymphabflussgebiet. Obwohl die Integration von 
modernen Techniken zu begrüßen ist, muss die Reduktion der Bestrahlungsdosis im elektiven Bestrahlungsvolumen aufgrund der schlechten Sensitivität des nodalen Stagings kritisch hinterfragt werden [4]. Eine alternative Anwendung der SIB-Technik könnte die lokale Intensivierung der RT bei Patienten mit erhöhtem Lokalrezidivrisiko sein, z. B. bei der Tumorkategorie cT4 oder potentiell knappem oder positivem zirkumferenziellen Resektionsrand (CRM) [5].

Nach sequentieller Anwendung von Kurzzeit-RT und FOLFOX-Chemotherapie wurde bei $9 \%$ der Patienten eine akute gastrointestinale Toxizität vom Grad $\geq 3$ beobachtet, was etwas weniger erscheint als nach FOLFOX-basierter präoperativer simultaner Langzeit-RT [6-8]. Eine Reduktion der FOLFOX-Dosis war allerding bei $24 \%$ der Patienten notwendig, was mindestens den Erfahrungen der oben zitierten Studien mit simultaner Therapie entspricht. Zudem konnte ein Patient aufgrund einer Chemotherapie-induzierten Sepsis einer kurativen Therapie nicht mehr zugeführt werden. Bei einem weiteren Patienten wird beschrieben, dass er aufgrund einer „new medical condition“ ebenfalls nicht operiert werden konnte; ob dies nicht auch eine Folge der präoperativen Therapie war, bleibt zu diskutieren. Inwieweit die sequentielle Therapie daher tatsächlich relevant die Toxizität reduziert, bleibt mit der aktuellen Studie unbeantwortet. Gerade die oben beschrieben modernen Bestrahlungstechniken könnten durch eine verbesserte Dünndarmschonung die Verträglichkeit einer Radiochemotherapie verbessern, simultan wie sequentiell appliziert [9].

Die Interpretation der onkologischen Ergebnisse wird durch eine fehlende Intention-to-Treat-Analyse erschwert: Drei Patienten, die nicht der Operation zugeführt werden konnten, wurden von der Analyse ausgeschlossen. Obwohl der primäre Endpunkt der Studie ein Downsizing der T-Kategorie war, wurde das klinische T-Stadium nur bei $<50 \%$ der Patienten mittels MRT ermittelt; bei $12 \%$ der Patienten wurde das Tumorstadium ausschließlich mit der klinischen Untersuchung erhoben. Das beschriebene T-Downsizing muss daher mit Vorsicht interpretiert werden. Bereits in früheren Studien wurde nach Kurzzeit-RT und verzögerter Operation ein Downstaging beobachtet, eine pCR wurde allerdings nur in $<10 \%$ der Patienten erzielt $[10,11]$. In der aktuellen Studie wurde aber bei $25 \%$ der Patienten eine pCR beobachtet, was ähnlich den Erfolgen der präoperativen Langzeit-RT ist [8].

\section{Fazit}

Traditionelle Zielvolumen- und Dosiskonzepte müssen auf die Kompatibilität mit intensivierter Systemtherapie überprüft werden. Trotz methodischer Mängel stellt die Studie von Myerson et al. daher ein attraktives Konzept zur Kombination von präoperative Kurzzeit-RT und volldosierter
FOLFOX-Chemotherapie dar, das aktuell bereits in einer Phase-III-Studie mit der konventionell fraktionierten Langzeit-RCHT verglichen wird [12].

Matthias Guckenberger, Zürich

\section{Einhaltung ethischer Richtlinien}

Interessenkonflikt M. Guckenberger gibt an, dass kein Interessenkonflikt besteht.

\section{Literatur}

1. Myerson RJ, Tan B, Hunt S, Olsen J, Birnbaum E, Fleshman J, Gao F, Hall L, Kodner I, Lockhart AC et al (2014) Five fractions of radiation therapy followed by 4 cycles of FOLFOX chemotherapy as preoperative treatment for rectal cancer. Int J Radiat Oncol Biol Phys 88:829-836

2. Bujko K, Nowacki MP, Nasierowska-Guttmejer A, Michalski W, Bebenek M, Kryj M (2006) Long-term results of a randomized trial comparing preoperative short-course radiotherapy with preoperative conventionally fractionated chemoradiation for rectal cancer. Br J Surg 93:1215-1223

3. Ngan SY, Burmeister B, Fisher RJ, Solomon M, Goldstein D, Joseph D, Ackland SP, Schache D, McClure B, McLachlan SA et al (2012) Randomized trial of short-course radiotherapy versus long-course chemoradiation comparing rates of local recurrence in patients with T3 rectal cancer: Trans-Tasman Radiation Oncology Group trial 01.04. J Clin Oncol 30:3827-3833

4. Evans J, Patel U, Brown G (2011) Rectal cancer: primary staging and assessment after chemoradiotherapy. Semin Radiat Oncol 21:169-177

5. Engels B, Platteaux N, Van den Begin R, Gevaert T, Sermeus A, Storme G, Verellen D, De Ridder M (2014) Preoperative intensitymodulated and image-guided radiotherapy with a simultaneous integrated boost in locally advanced rectal cancer: report on late toxicity and outcome. Radiother Oncol 110:155-159

6. Gerard JP, Azria D, Gourgou-Bourgade S, Martel-Laffay I, Hennequin C, Etienne PL, Vendrely V, Francois E, de La Roche G, Bouche $\mathrm{O}$ et al (2010) Comparison of two neoadjuvant chemoradiotherapy regimens for locally advanced rectal cancer: results of the phase III trial ACCORD 12/0405-Prodige 2. J Clin Oncol 28:1638-1644

7. Aschele C, Cionini L, Lonardi S, Pinto C, Cordio S, Rosati G, Artale S, Tagliagambe A, Ambrosini G, Rosetti P et al (2011) Primary tumor response to preoperative chemoradiation with or without oxaliplatin in locally advanced rectal cancer: pathologic results of the STAR-01 randomized phase III trial. J Clin Oncol 29:2773-2780

8. Rodel C, Liersch T, Becker H, Fietkau R, Hohenberger W, Hothorn T, Graeven U, Arnold D, Lang-Welzenbach M, Raab HR et al (2012) Preoperative chemoradiotherapy and postoperative chemotherapy with fluorouracil and oxaliplatin versus fluorouracil alone in locally advanced rectal cancer: initial results of the German CAO/ARO/AIO-04 randomised phase 3 trial. Lancet Oncol 13:679-687

9. Garofalo M, Moughan J, Hong T, Bendell J, Berger A, Lerma F, Lee R, Anne P, Sharma N, Crane C (2011) RTOG 0822: a phase $\mathrm{H}$ study of preoperative (PREOP) chemoradiotherapy (CRT) utilizing IMRT in combination with capecitabine $(\mathrm{C})$ and oxaliplatin (O) for patients with locally advanced rectal cancer. Int J Radiat Oncol 81:3-4 
10. Beppu N, Matsubara N, Noda M, Yamano T, Doi H, Kamikonya N, Yamanaka N, Yanagi H, Tomita N (2014) The timing of surgery after preoperative short-course S-1 chemoradiotherapy with delayed surgery for T3 lower rectal cancer. Int J Colorectal Dis. Epub 2014 Aug 28

11. Pettersson D, Holm T, Iversen H, Blomqvist L, Glimelius B, Martling A (2012) Preoperative short-course radiotherapy with delayed surgery in primary rectal cancer. Br J Surg 99:577-583
12. Nilsson PJ, van Etten B, Hospers GA, Pahlman L, van de Velde CJ, Beets-Tan RG, Blomqvist L, Beukema JC, Kapiteijn E, Marijnen CA et al (2013) Short-course radiotherapy followed by neoadjuvant chemotherapy in locally advanced rectal cancer-the RAPIDO trial. BMC Cancer 13:279 